\title{
UWB-WBAN Radio Channel Characteristics between the Endoscope Capsule and On-body Antenna
}

\author{
Mariella Särestöniemi ${ }^{1}$, Carlos Pomalaza Raez ${ }^{2}$, Markus Berg ${ }^{1}$, Chaïmaâ Kissi ${ }^{3}$, Matti \\ Hämäläinen ${ }^{1}$, Jari Iinatti ${ }^{1}$ \\ ${ }^{1}$ Centre for Wireless Communications, University of Oulu \\ ${ }^{2}$ Department of Electrical and Computer Engineering, Purdue University \\ ${ }^{3}$ Electronics and Telecommunication Systems Research Group, National School of Applied \\ Sciences (ENSA), Ibn Tofail University
}

\begin{abstract}
This paper presents a study on the radio channel characteristics between an endoscope capsule and an on-body antenna in different parts of the small intestine with different onbody antenna location options. The study is conducted using finite integration technique based electromagnetic simulation software CST and one of its anatomical voxels. An endoscope capsule model with a dipole antenna is set inside different areas of the small intestine of the voxel model. A recently published highly-directive on-body antenna designed for on-in-body communications is used in the evaluations. Different rotation angles of the capsule are also considered both with a layer model and a voxel model. It is found that radio channel characteristics vary remarkably depending on the antenna location in the small intestine and location of the on-body antenna. Thus, the on-body antennas should be located carefully to ensure coverage over the whole intestine area. However, the path loss does not only depend on the distance between a capsule and the on-body antenna but also on the tissues between the capsule and onbody antennas. Furthermore, orientation of the capsule has also strong impact when linearly polarized antennas are used.
\end{abstract}

Keywords: capsule endoscopy, directive antenna, gastrointestinal monitoring, implant communications, ultra wideband, wireless body area networks

\section{Introduction}

Capsule endoscopy has become a popular method to investigate the gastrointestinal (GI) tract area due to its several advantages: it is reliable, painless, and comfortable way to examine especially the small intestine (SI) area, which is not easily reached with the conventional endoscopy techniques. In the capsule endoscopy, the patient just swallows a small pill, which contains a camera and transmitter, and wears a monitoring device on the waist where the data from the capsule is transmitted. [1]-[2]

Different techniques are used to transmit the data from the capsule to the on-body device. Ultra wideband (UWB) has recently become an attractive alternative for the communication link since it enables reliable and high-data rate data transfer with low power consumption and simple electronics. Besides, large bandwidth enables high resolution for the images. [3]-[5] The frequency bandwidth determined by the IEEE 802.15.6 standard for Wireless Body Area Networks (WBAN) [6] is 3.1 - $10.6 \mathrm{GHz}$. The propagation losses in the tissues increase as the frequency increases. Thus, the 
lower part of the UWB band is usually considered for the capsule endoscopy application.[3]-[5]

Smooth design of transceivers require deep knowledge of the channel characteristics and propagation within the tissues. Thus, there are several UWB channel models and propagation studies presented in the literature for wearable and implant communication systems in the human abdomen area [7]-[17]. Furthermore, the research in antenna design, both for capsule antennas as well as for on-body receiver antennas, has been active recently [18]-[25]. Channel characteristics between the capsule antenna and on-body antenna is studied e.g. in [3],[5], [7], [24]. In-body power distribution in the abdominal area was studied in detail in [7]. It was shown that the power inside the abdomen area is distributed depending on the tissues and antenna locations. Even challenging locations in the small intestine area can be reached with appropriate onbody antenna locations

This paper is continuation of [7] by presenting UWB-WBAN channel characteristics between the endoscope capsule and recently published high-directive on-body antennas in different parts of the SI. Two different on-body antenna location options, both suitable for capsule endoscopy, are considered. Frequency and time domains are considered. Furthermore, the impact of the capsule's rotation angle is studied on the channel characteristics both with a layer model and the voxel model.

The paper is organized as follows: Section II describes the study case, i.e., voxel model, antennas, and antenna locations. Section III presents the results for channel characteristics between the endoscope capsule and on-body antenna as well as the impact of the rotation angles. Conclusions are given in Section IV.

\section{Study Case}

Since this paper is continuation of the research presented in [7], the study case can be studied more in detail in [7]. However, this section briefly repeats the most essential information for ease reading.

\subsection{Voxel model}

Simulations were conducted using the electromagnetic simulation software CST MicroWave Studio [27], which is based on finite integration technique (FIT). CST provides several voxel models, among which we selected an anatomical voxel model Laura, presented in Fig, 1a. Laura corresponds to lean female body with resolution of $1.87 \mathrm{~cm} \times 1.87 \mathrm{~cm} \times 1.87 \mathrm{~cm}$. Cross-section of the voxel model's abdomen area on the navel line is shown in Fig 1b. 


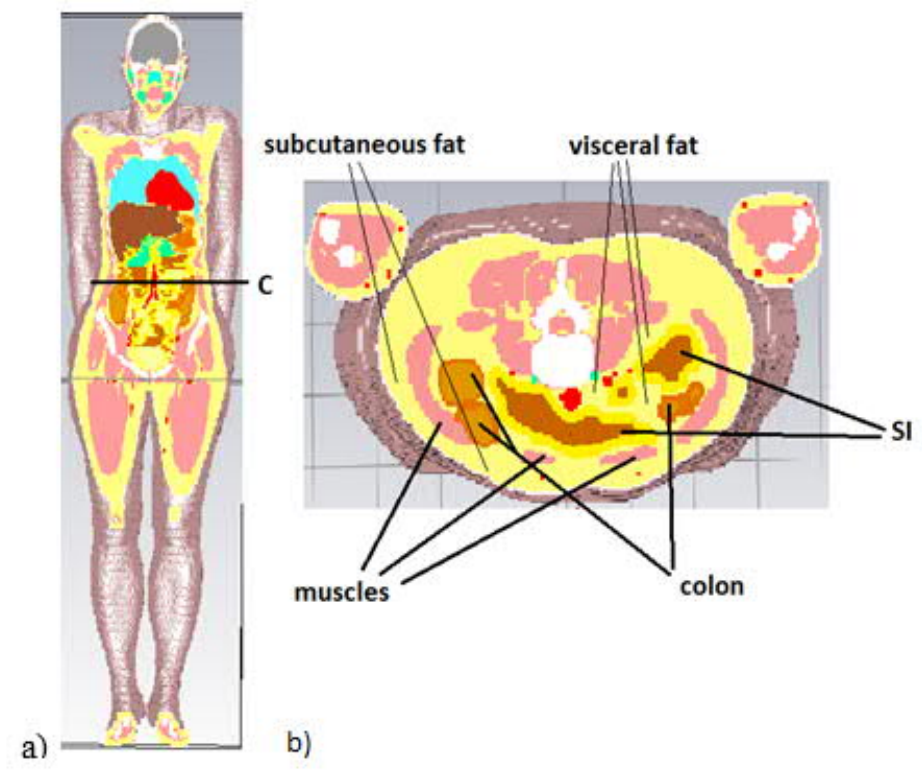

Fig. 1 a) Anatomical voxel model Laura, b) cross section of the abdomen area presenting the subcutaneous and visceral fat.

\subsection{On-body antenna and antenna locations}

In this study case, we use a cavity-backed low-band UWB antenna designed for on-in-body communications. The antenna is presented in Fig. 2 [18]. This antenna is designed to work in the frequency band $3.75-4.25 \mathrm{GHz}$. Two different antenna location options were considered, both of them are suitable for monitoring purposes [7]. The antenna location options are presented in the Figs 3 a-b.

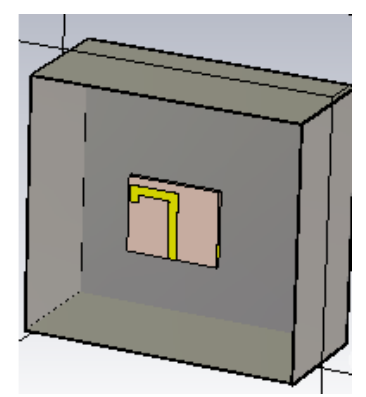

Fig. 2 A cavity-backed low-band UWB on-body antenna designed for in-body communications. 

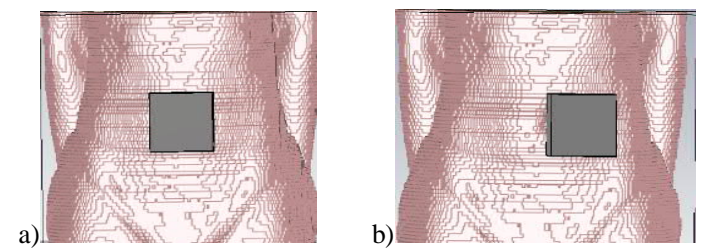

Fig. 3 Location of the on-body antenna a) on the navel, b) on the side.

\subsection{Capsule model}

In this study, we use a simplified capsule model, in which a simple dipole antenna is embedded in the plastic capsule shell, as presented in Figs 4 a-b, respectively. The capsule itself has realistic dimensions: $11 \mathrm{~mm}$ x $25 \mathrm{~mm}$, corresponding to the size of the commercial capsules nowadays. The dipole antenna is omnidirectional and it is designed to work at the frequency $4 \mathrm{GHz}$. The reflection coefficient $\mathrm{S} 11$ and the radiation pattern of the dipole antenna are presented in Fig $5 \mathrm{a}-\mathrm{b}$, respectively.

a)

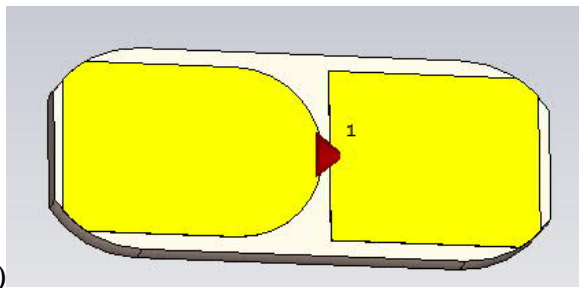

Fig. 4 a) dipole antenna inside the capsule, b) capsule shell

b)

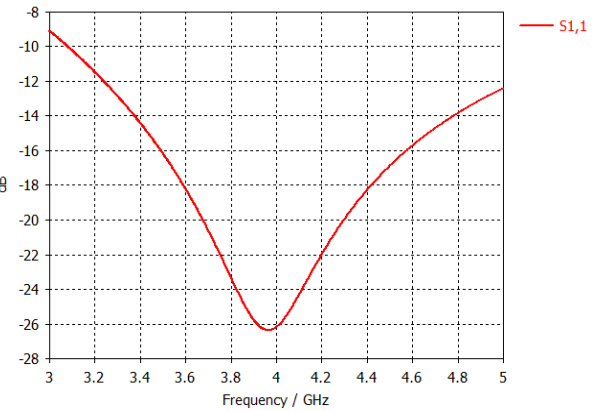

Fig. 5. a) S11 and b) radiation pattern of the antenna.
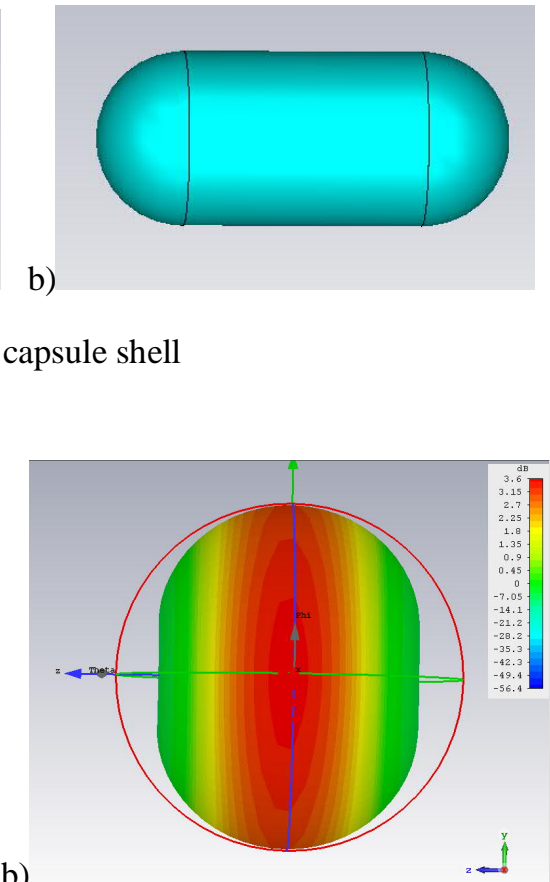


\section{$3 \quad$ Layer Model}

The layer model used in this study is presented in Fig. 6a. Tissue thicknesses in the layer model correspond to the thicknesses of the voxel model in the cross-section $\mathrm{C}$ in Fig. 1 with antenna location option 2 shown in Fig. 6b. The location of the capsule in the small intestine is included in the voxel model as well. The thicknesses of the different layers are summarized in Table I. One should note that the thicknesses of different layer models may vary depending which part of the abdomen is considered. Table I also summarizes the dielectric properties of these tissues to describe different propagation properties [28].

a)

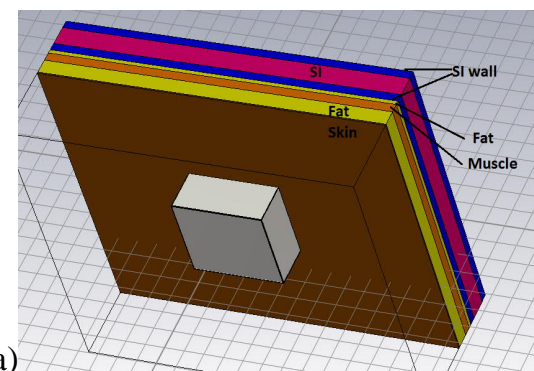

b)

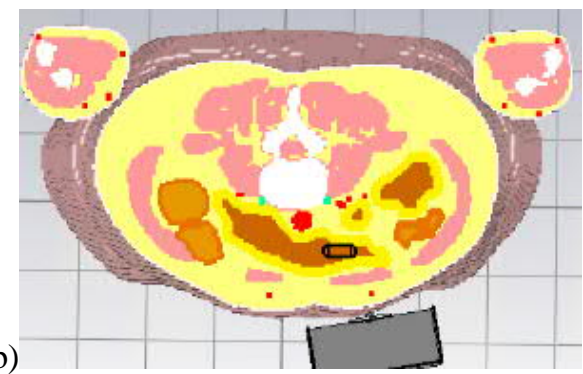

Fig. 6 a) Layer model designed using the dimensions based on b) antenna location 2 and cross-section $\mathrm{C}$.

Table I Layer model dimension and dielectric properties of tissues at $4 \mathrm{GHz}$.

\begin{tabular}{|l|l|l|l|}
\hline & $\begin{array}{l}\text { Thickness } \\
{[\mathrm{mm}]}\end{array}$ & Permittivity & Conductivity \\
\hline Skin & 1.4 & 36.6 & 2.34 \\
\hline $\begin{array}{l}\text { Fat subcutane- } \\
\text { ous }\end{array}$ & 15 & 5.13 & 0.183 \\
\hline Muscle & 9 & 50.8 & 3.01 \\
\hline Fat (visceral) & 4 & 5.13 & 0.183 \\
\hline SI wall & 8 & 50.82 & 3.105 \\
\hline SI content & 20 & 51.7 & 4.62 \\
\hline
\end{tabular}

\section{Simulation results}

\subsection{Layer model vs. voxel model comparison}

This section compares the channel characteristics obtained with the layer model and the voxel model. The frequency domain results - path loss - and time domain results - impulse responses (IR) - obtained by performing inverse fast Fourier transform (IFFT) for the S21, for the layer model and voxel model are presented in 
Fig.s 7 a-b, respectively. As one can note, there is clear difference between the results obtained using the layer model and the voxel model both in frequency and time domains. In the frequency range of interest, i.e. at $3.75-4.25 \mathrm{GHz}$, the channel parameters S21, which corresponds to path loss, is even $10 \mathrm{~dB}$ lower with the layer model than with the voxel model. In the time domain, the levels of the first three peaks are $10 \mathrm{~dB}$ lower with the layer model.

The reason for this tendency is conjectured to be due to two reasons: 1) the pixels of the voxel model has more space between the antenna and the skin, which improves the antenna performance [18] and hence may improve the channel characteristics as explained in [14]. 2) The layer model mainly takes account the simple direct propagation through the tissues whereas the voxel model includes the impact of more complex indirect propagation paths, e.g. through the fat layer, as explained in [7], [8].

a)

용

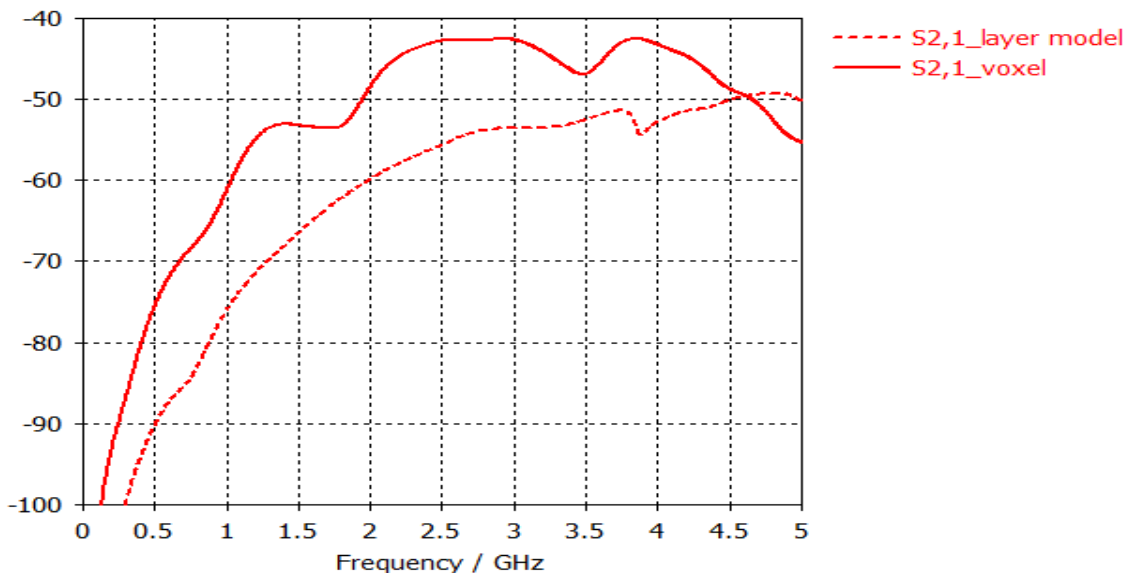

b)

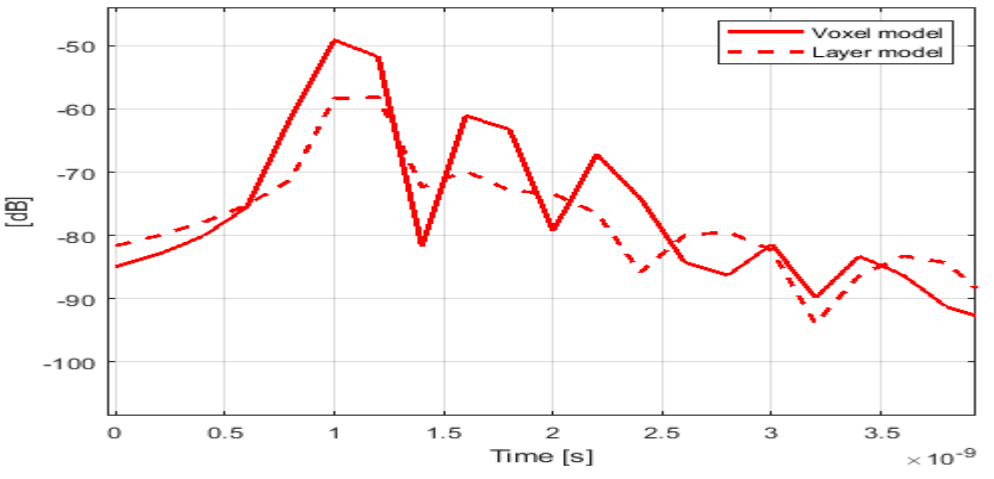

Fig. 7 a) Frequency and b) time domain comparison between the voxel model and layer model results. 


\subsection{On-body antenna location 1}

In this section, we present channel characteristics between the capsule and the lowband UWB cavity-backed on-body antenna at the cross-section A (see Fig. 1) with different antenna location options. The channel characteristics are evaluated at three different capsule locations, which are presented in Fig 8. In the option "a", the capsule is in the middle of the SI at the cross-section B, i.e., the distance between the on-body antenna and the capsule is the smallest. In the capsule location option "b", the antenna is on the front-left part of the SI, and in the capsule location option "c", the antenna is on the right back part of the SI. These capsule location options are chosen to cover the most interesting parts of the SI in terms of in-body power distribution studies presented in [7].

a)

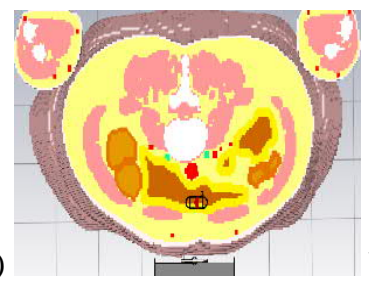

b)

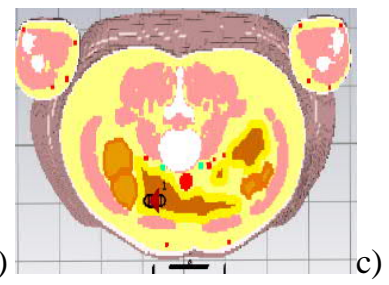

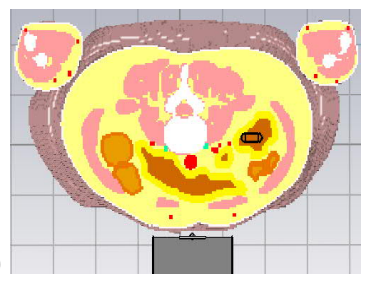

Fig. 8 Locations of the capsule a) in the middle of the SI's front part, b) in the left side of the SI's front part, c) in the right side of the SI's back part.

The frequency and time domain channel characteristics are presented in Fig. 9 a-b, respectively. Evidently, the path loss is lowest as the capsule is located in the middle of the SI (case a), since the distance between the capsule and the on-body antenna is smallest and since the part of the signal may travel without passing the muscle layer, as explained in [7] and [8]. Instead, the path loss difference between the cases $b$ and $c$ is surprisingly small in the frequency range of interest, although "c" is clearly further from the on-body antenna than in the case "b". Actually at $3.8 \mathrm{GHz}$, the path loss in location "b" is even higher than at point "c". Since there are no remarkable differences in the antennas' radiation patterns toward point "b" and "c", as presented in [18], the reason for this phenomenon can be found from the propagation paths. For the point " $\mathrm{c}$ ", there is a propagation path option through the fat layer, in which the losses are minor, as explained in [7], [8], [12],[13]. Instead, for the point "b", most of the signal needs to travel through the small intestine tissue, in which the losses are high.

In the time domain, as the IFFT is performed for the whole bandwidth, the differences are clearer. Besides, the shapes of the IRs differ from each other remarkably. The further away the capsule is from the on-body antenna, the richer is the IR with larger number of significant propagation paths, which appear as the signal propagates inside the body avoiding the most challenging tissues.

The path loss in the case of point "c" is excessive to provide enough strong communications link between the capsule and the on-body antenna. Thus, we need to evaluate the channel characteristics when the on-body antenna is located on the side 
of the abdomen as well. The next subsection channel characteristics with the antenna location option 2 .
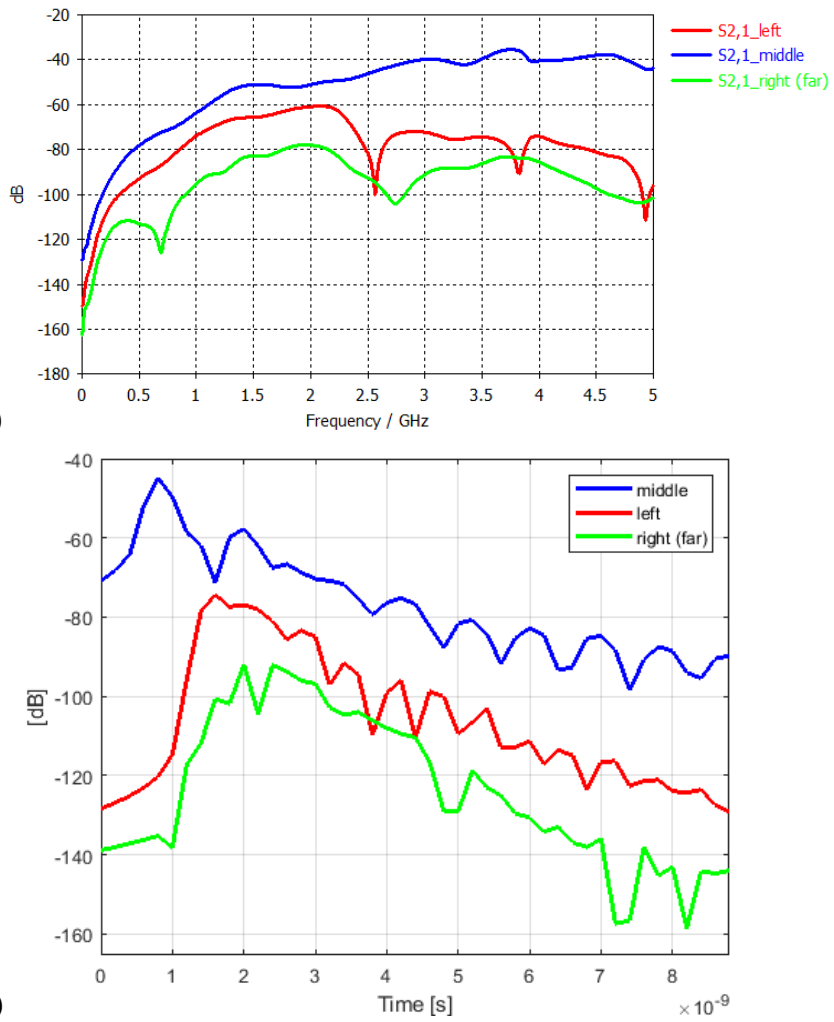

Fig. 9. a) Path losses and b) IRs of the capsule - on-body antenna link at the antenna location option 1.

\subsection{On-body antenna location 2}

Next, we evaluate the channel characteristics with the antenna location option 2. Capsule locations are presented in Fig $10 \mathrm{a}-\mathrm{c}$ as a) left (the furthest possible location), b) middle (the second furthest location) and c) in the right side of the SI's back part. The frequency and time domain channel characteristics are presented in Figs $11 \mathrm{a}-\mathrm{b}$. The channel is naturally at strongest in the capsule location " $b$ ", since the distance between the capsule and the on-body antenna is the smallest of these presented cases. The channel at capsule location "a" is stronger than in the location "c", although the distance between the capsule and the on-body antenna is larger in the case of "a" The path loss is even $10 \mathrm{~dB}$ higher at the location "c" than in the location "a" at $3.75 \mathrm{GHz}$. Instead, at $4-4.25 \mathrm{GHz}$, the path loss is same. In the time domain, the difference is the strongest for first two peaks. 
The channel in the point "a" is stronger than in the point "c" due to the more favorable propagation paths towards the on-body antenna. For instance in the case of point "a", according to the voxel model's cross-section, the signal can propagate through the fat layer without passing the muscle layer, which is known to be the among the worst tissues for the propagation. Instead, in the point "c", the signal has to pass the muscle layer before reaching the on-body antenna, which reduced the power more. These inbody power distribution and propagation path issues are explained more in detail in [7].

The path loss with the antenna location option 2 in the point "c" is still high, though slightly lower than with the antenna location option 1. To ensure good communication link between the capsule and the on-body antenna in the location "c", one receiving on-body antenna should be placed further from the navel area towards sides of the body. Besides, it is essential to have more than one on-body antenna in the capsule endoscopy operations.

a)

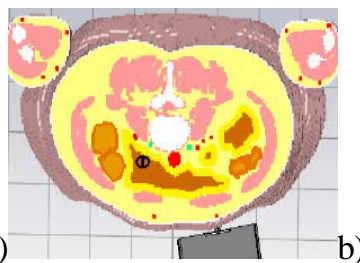

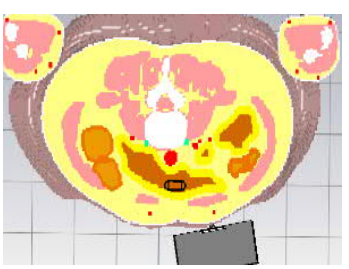

c)

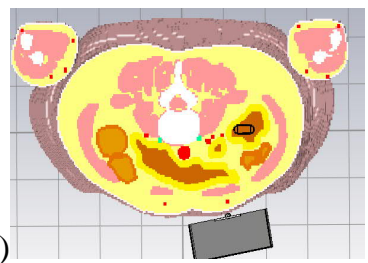

Fig. 10 Locations of the capsule a) in the middle of the SI's front part, b) in the left side of the SI's front part, c) in the right side of the SI's back part.

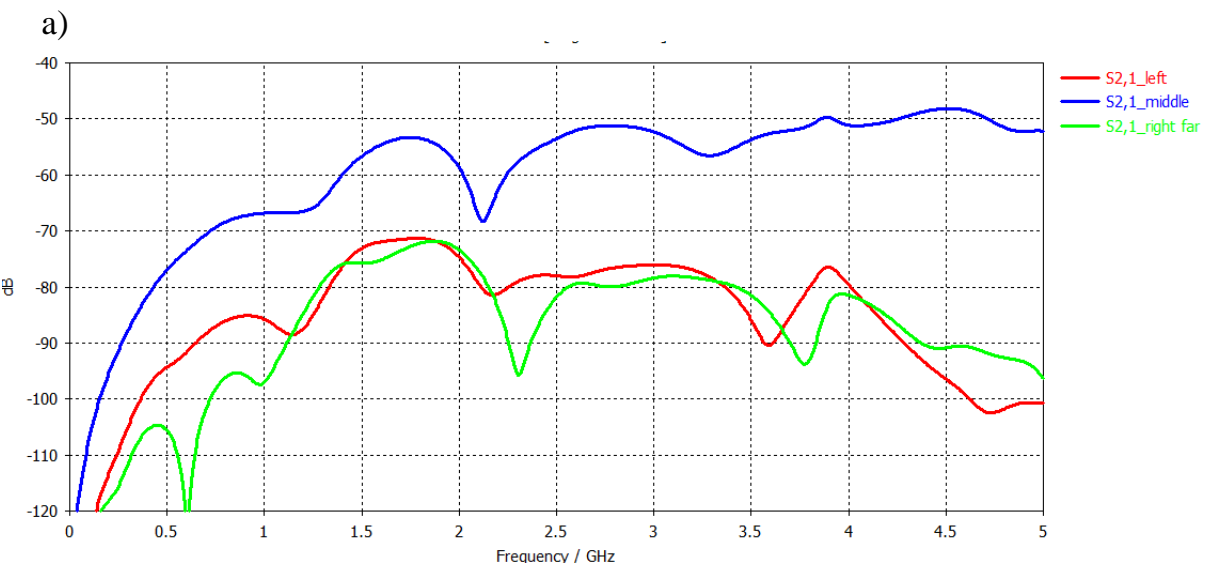




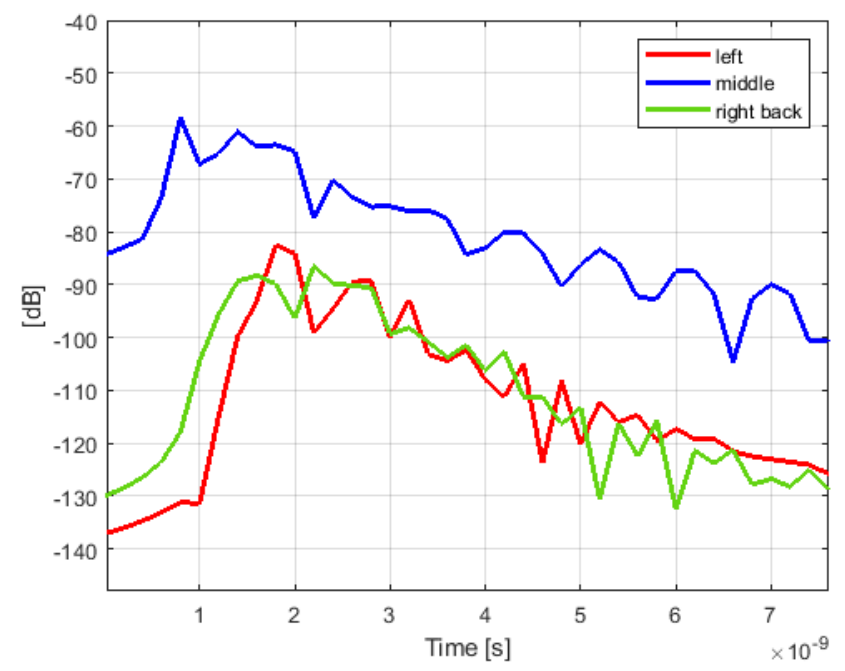

Fig. 11. a) Path losses and b) IRs of the capsule - on-body antenna link at the antenna location option 2.

\subsection{Impact of the rotation on the channel characteristics}

\section{Layer model results}

In this subsection we present the impact of the capsule's rotation angle on the channel characteristics. Since the antennas are linerly polarized, the rotation angle is assumed to have a clear impact on the power loss.

First, the impact is evaluated with the layer model for simplicity. We evaluated the rotation angles $0^{\circ}, 45^{\circ}$ and $90^{\circ}$, as shown in Fig.s 13 a-c. As one can note, rotation of the capsule impacts clearly on the channel characteristics both in frequency and time domains. Naturally, the difference is largest betweeen the rotation angles $0^{\circ}$ and $90^{\circ}$. The path loss difference is maximum $13 \mathrm{~dB}$ within the frequency range of the interest. The smallest path loss difference is $4 \mathrm{~dB}$ at $4 \mathrm{GHz}$. In time domain, the largest difference can be seen in the level and the shape of the IR's main peaks. The level difference is up to $8 \mathrm{~dB}$ in the main peaks.
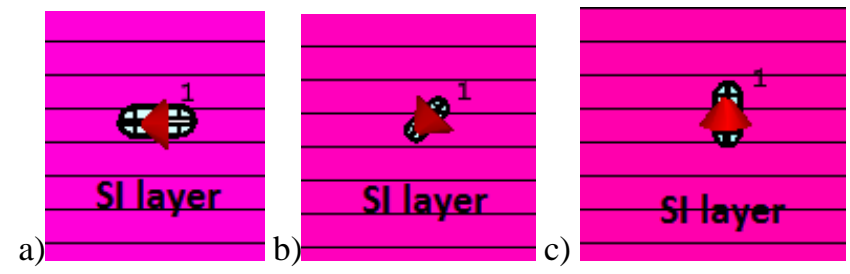

Fig. 12. Rotation angles of the capsule a) $0^{\circ}$, b) $45^{\circ}$, and c) $90^{\circ}$ with layer model 
a)

웅

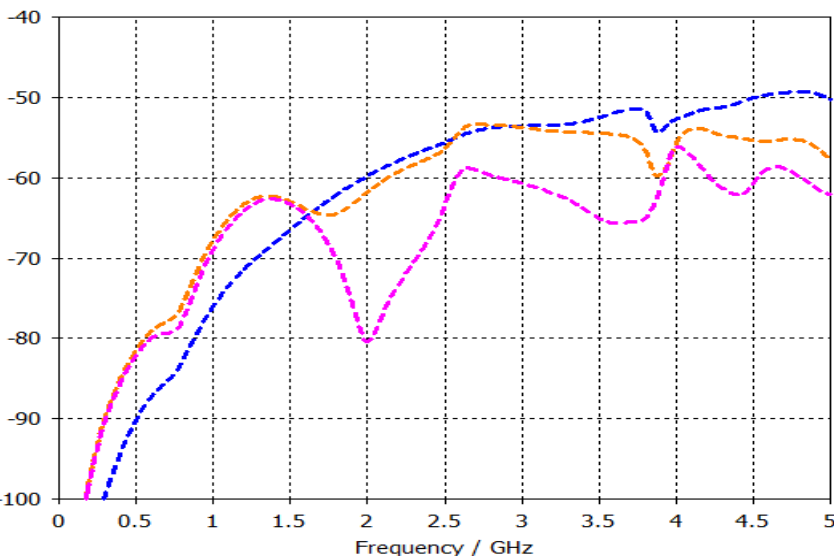

- - S2,1_layer model 0 - - S2,1_layer model 45 - - 52,1 layer model 90.

b)

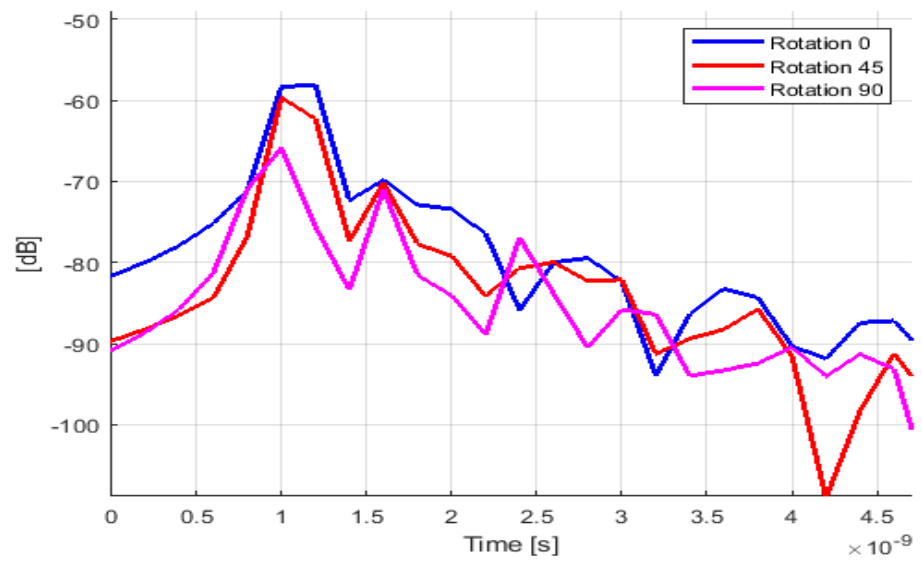

Fig. 13 Channel characteristics between the capsule - on-body antenna link with different rotation angles of the capsule in a) frequency domain and b) time domain, layer model results.

\section{Voxel model results}

The impact of the rotation is evaluated for the case with the on-body antenna and capsule location presented in Fig 6b. The thickness of the tissues just below the antenna in this scenario is the same as with the layer model. We evaluated the rotation angles $0^{\circ}, 45^{\circ}$ and $90^{\circ}$, which are presented in vertical cross-sections in Figs 14a-c.

Fig. 15a-b present the channel characteristics with different rotation angles of the capsule in frequency and time domains, respectively. As one can note from Fig 15a, there is only minor differences in the path losses between the rotation angle cases $0^{\circ}$ and $45^{\circ}$ in the frequency range $2-5 \mathrm{GHz}$. Instead, with the rotation angle $90^{\circ}$ the path loss is 10-17 dB lower than with rotation angles 0 and 45 in the frequency band of the 
interest. Similar tendency can be found in the main peaks of the IRs opresented in Fig 15b: the level of the main peak is approximately same for the cases with the rotation angle 0 and 45, whereas the main peak obtained with the rotation angle $90^{\circ}$ is $6 \mathrm{~dB}$ lower. Interestingly, the level of the second peak is the highest for the case with the rotation angle $90^{\circ}$. In general, the rotation angle changes clearly the shape of the IR after the main peak: the timing, width, and level of the side peaks very remarkably. Hence, the rotation of the capsule affects on the propagation path which the signal choses.

When comparing the impact of the rotation obtained with layer model and voxel model, one can note similarities only in the path loss behaviour. Naturally, the level difference is same as explained in subsection 3.1 In time domain, there are clear differences between the side peaks obtained using the layer model and voxel model. It is obvious that the rotation affects on the propagation paths, which can not be investigated accurately with the simple layer models. The impact of the rotation on the channel characteristics will be studied more in detail with the voxel models in the extension of this paper.
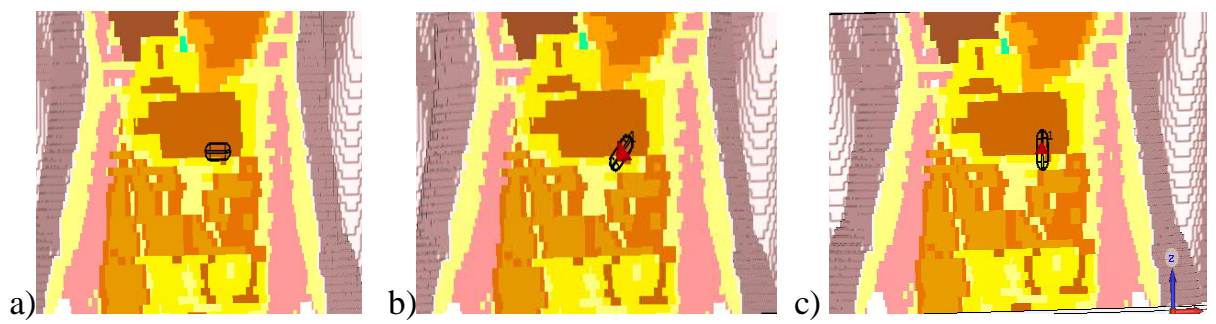

Fig. 14 Rotation angles of the capsule a) $0^{\circ}$, b) $45^{\circ}$, and c) $90^{\circ}$.

a)

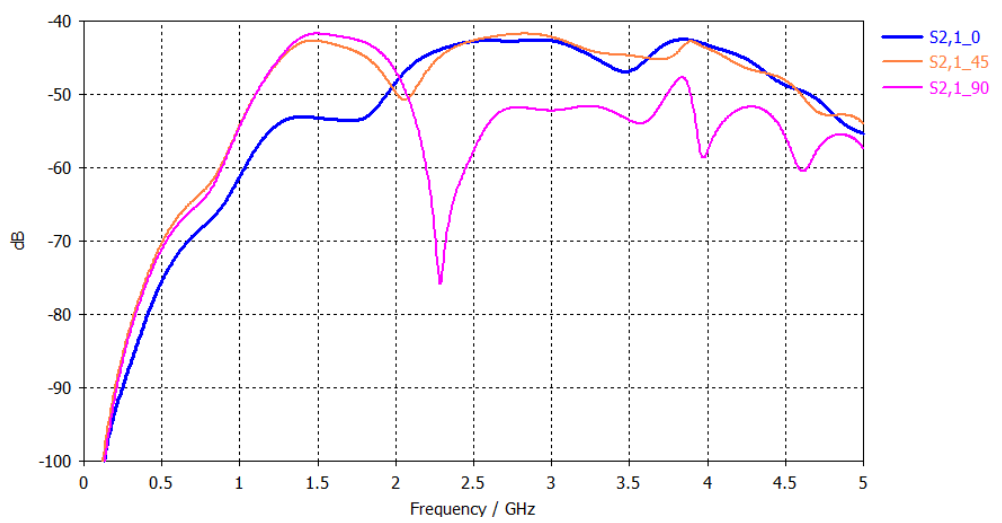


b)

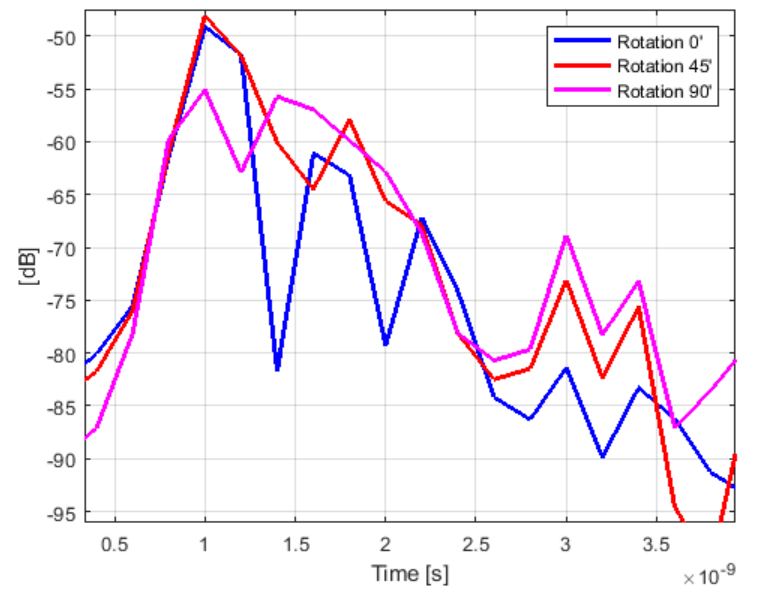

Fig. 15 Channel characteristics between the capsule - on-body antenna link with different rotation angles of the capsule in a) frequency domain and b) time domain.

\section{Conclusions}

This paper presents a study on the radio channel characteristics between the capsule endoscope and the high-directive on-body antenna in different parts of the small intestine with different on-body antenna locations. The study was conducted using finite integration technique based electromagnetic simulation software CST and its anatomical voxel model. A capsule endoscope with a dipole antenna was set inside different areas of the small intestine of the voxel model. The impact of the different rotation angles of the capsule on the channel characteristics was also evaluated both with a layer model and a voxel model. It is found that the radio channel characteristics varied remarkably depending on the capsule location in the small intestine and location of the on-body antennas. Thus, the locations of on-body antennas should be carefully selected to ensure coverage over the whole intestine area. Furthermore, orientation of the capsule had also clear impact on the channel characteristics when linearly polarized antennas are used. The largest difference in the path losses of different cases is $16 \mathrm{~dB}$. In the main peaks of the IR's the difference is only $6 \mathrm{~dB}$ but in the side peaks, the difference is larger.

The results presented in this study provide insight on the channel characteristics, which could be obtained when a highly directive on-body UWB antenna is used. In this study, we used a simple dipole antenna on the capsule. The path loss could be diminished even more with circular-polarized antennas, either on the body or inside the capsule. Our future target is to evaluate channel characteristics with different 
highly-directive antennas and also with more realistic capsule structure. Furthermore, the impact of the rotation is studied more in detail.

\section{Acknowledgements}

This research has been financially supported by the project WBAN Communications in the Congested Environments and in part by Academy of Finland 6Genesis Flagship (grant 318927). Ilkka Virtanen, Timo Mäkinen, and Jari Sillanpää from University of Oulu deserve acknowledgement for their help to enable the exhaustive simulations. Dr. Marko Sonkki from CWC, University of Oulu, is acknowledged for his participation on the on-body antenna design.

\section{References}

1. G. Ciuti; A. Menciassi; P. Dario, “ Capsule Endoscopy: From Current Achievements to Open Challenges", IEEE Reviews in Biomedical Engineering, vol.4, pp. 59 - 72, 2011.

2. H. Neumann, L. C. Fry, A. Na"gela, and M. F. Neurath ; "Wireless capsule endoscopy of the small intestine: a review with future directions"; Current Opinion in Gastroenterology; Vol 30; Issue 5; pp 463-471, 2014.

3. P. Ara, K. Yu, S. Cheng, E. Dutkiewicz, M. C. Heimlich, "Human Abdomen Path-Loss Modeling and Location Estimation of Wireless Capsule Endoscope Using Round-Trip Propagation Loss,” IEEE Sensor Journal, Vol. 18, No. 8, 2018

4. R. Chavez-Santiago, J. Wang, I. Balasinham, "The Ultra Wideband Capsule Endoscope," Internatiomal Conf. on Ultra Wideband, 2013.

5. S. Stoa, R. Chavez-Santiago, I. Balasinham, "An ultra wideband communication channel model for capsule endoscopy," ISABEL2014

6. IEEE Standard for Local and metropolitan area networks_Part 15.6: Wireless Body Area Networks, pp. IEEE Std 802.15.6-2012, pp. 1 - 271, 2012.

7. M. Särestöniemi, C. Pomalaza-Raez, M. Berg, C. Kissi, M. Hämäläinen. J. Iinatti, "InBody Power Distribution for Abdominal Monitoring and Implant Communications Systems," accepted to be published in ISWCS, September 2019

8. M. Särestöniemi, C. Pomalaza-Raez, M. Berg, C. Kissi, M. Hämäläinen. J. Iinatti, "Fat in the Abdomen as a Propagation Medium in WBAN Applications," submitted to Bodynets2019.

9. A. Khaleghi, R. Chávez-Santiago, X. Liang, I. Balasingham, V. C. M. Leung, T. A. Ramstad, "On ultra wideband channel modeling for in-body communications", Proc. IEEE Int. Symp. on Wireless Pervasive Computing (ISWPC), May 5-7, 2010.

10. A. Teshome, B. Kibret, D. T. H. Lai, "A Review of Implant Communication Technology in WBAN, Progresses and Challenges," IEEE Reviews in Biomedical Engineering, 2018.

11. P. Leelatien, K. Ito, K. Saito, M. Sharma, A. Alomainy, "Channel Characteristics and Wireless Telemetry Performance of Transplanted Organ Monitoring System Using Ultrawideband Communication," IEEE Journal of Electromagnetics, RF and Microwaves in Medicine and Biology, 2018.

12. N. B. Asan, et al. Intra-body Microwave Communication Through Adipose Tissue, healthcare Technology Letter, 2017.

13. N. B. Asan et al. Characterization of Fat Channel for intra-Body Communication at RBand Frequencies, MDPI Sensors, 2018. 
14. M. Särestöniemi; C. Kissi;C. Pomalaza-Raez; T. Kumpuniemi ;M. Sonkki; S. Myllymäki ; M. Hämäläinen ; and J. Iinatti, "Measurement and simulation based study on the UWB channel characteristics on the abdomen area", ISMICT 2019.

15. M. Särestöniemi; C. Kissi; C. Pomalaza-Raez ; M. Hämäläinen; and J. Iinatti, "Impact of the antenna-body distance on the UWB on-body channel characteristics", ISMICT 2019.

16. M. Särestöniemi; C. Kissi; C. Pomalaza Raez;. M. Hämäläinen and J. linatti, ”Propagation and UWB channel characteristics on human abdomen area," EUCAP 2019.

17. J. Li, Z. Nie, Y. Liu, L. Wang, Y. Hao, "Characterization of In-Body Radio Channels for Wireless Implants," IEEE Sensors Journal, 2017.

18. C. Kissi; M. Särestöniemi; C. P-. Raez; M. Sonkki; and M. N. Srifi, "Low-UWB directive antenna for Wireless Capsule Endoscopy localization", BodyNets2018

19. C. Kissi ; M. Särestöniemi ; T. Kumpuniemi ; M. Sonkki ; S. Myllymäki ; M. N. Srifi and C. P-. Raez, “Low-UWB Receiving antenna for WCE Localization”, ISMICT 2019 conference.

20. C. Kissi ; M. Särestöniemi ; T. kumpuniemi ; M. Sonkki ; S. Myllymäki ; M. N. Srifi and C. P-. Raez, "Low-UWB Antennas in Vicinity to Human Body", ISMICT 2019 conference.

21. C. Kissi ; M. Sarestonierni ; C. P-. Raez; M. Sonkki ; S. Myllymäki ; M. N. Srifi and H. Jantunen, "High-Directivity Antenna for Low-UWB Body Area Networks Applications", International Symposium on Advanced Electrical and Communication Technologies (ISAECT), pp. 1-6, 2018.

22. Z. Bao, "Comparative Study of Dual-Polarized and Circularly-Polarized Antennas at 2.45 GHz for Ingestible Capsules," Transaction on Antennas and Propagation,

23. W. Lei, Y-X Guo, "Design of Dual-Polarized Wideband Conformal Loop Antenna for Capsule Endoscopy Systems,"2017

24. K. Y. Yazdandoost, "Antenna for Wireless Capsule Endoscopy at Ultra Wideband Frequency," International Symposium on Personal, Indorr, and Mobile Radio Communications (PIMRC) 2017.

25. S. H. Lee; J. Lee; Y. J. Yoon; S. Park; C. Cheon; K. Kim; and S. Nam, "A Wideband Spiral Antenna for Ingestible Capsule Endoscope Systems: Experimental Results in a Human Phantom and a Pig", IEEE Transactions on Biomedical Engineering, Vol. 58, pp. 1734 $1741,2011$.

26. CST Microwave Studio, [Online]. Available: http://www.cst.com

27. https://www.itis.ethz.ch/virtual-population/tissue-properties/database 